\title{
Top-quark production measurements
}

\author{
Markus Cristinziani*i \\ On behalf of the ATLAS, CDF, CMS, D0 and LHCb Collaborations \\ Physikalisches Institut, University of Bonn, Germany \\ E-mail: cristinz@uni-bonn.de
}

Recent measurements of top-quark production at hadron colliders are reviewed. The inclusive top-quark pair production is determined at four centre-of-mass energies at Tevatron and LHC with experimental uncertainties that are close to the uncertainties in theoretical calculations at next-tonext-to-leading order in QCD. Several differential measurements are performed and compared to simulation. Production of single top quarks is studied in the three different production channels. Top-quark pair production with neutral and charged vector bosons has been observed by the LHC experiments. Finally, production of additional heavy flavour quark pairs $(b \bar{b}, t \bar{t})$ is studied or searched for.

XXVII International Symposium on Lepton Photon Interactions at High Energies 17-22 August 2015

Ljubljana, Slovenia

${ }^{*}$ Speaker.

${ }^{\dagger}$ Supported by European Research Council grant ERC-CoG-617185 


\section{Introduction}

Top quarks are the elementary particles with the largest mass and are therefore subject of intensive study at hadron colliders, the proton-antiproton collider Tevatron, operated until 2011 at up to $1.96 \mathrm{TeV}$ centre-of-mass energy, and the Large Hadron Collider (LHC) at CERN, operated at energies of 7, 8 and, more recently, $13 \mathrm{TeV}$.

From the large number of available results, I have chosen to illustrate the discussion exclusively with results that have been made public in the last twelve months by the ATLAS, CDF, CMS, D0 and LHCb Collaborations, effectively covering the full spectrum of studies of top-quark pair production, inclusive and differential, single top-quark production as well as associated production with vector bosons and flavoured quarks. For an overview of recent developments in the theory of top quarks see Ref. [1]. Properties of top quark are presented separately [2].

\section{Inclusive $t \bar{t}$ production cross section}

Top-quark pair production at Tevatron is dominated by $q \bar{q}$ annihilation, while it is dominated by gluon-gluon fusion at the LHC and therefore measurements at both colliders are complementary and allow to test different aspects of perturbative QCD calculations, which are now known at full next-to-next-to-leading order (NNLO) including gluon resummation.

Using the full Tevatron Run II dataset, the D0 Collaboration measures the inclusive $t \bar{t}$ cross section [4] to be $\sigma_{t \bar{t}}=7.73 \pm 0.13$ (stat.) \pm 0.55 (syst.) pb in final states with one or two leptons (electrons or muons), exploiting $b$-tagging information. In the $\ell+$ jets channel additional topological variables are employed (Fig. 1).

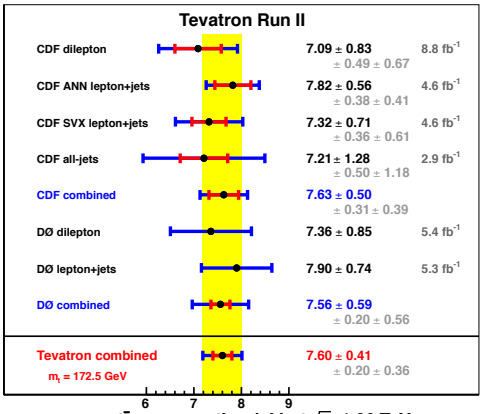

$\mathrm{pp} \rightarrow \mathrm{tt}$ cross section $(\mathrm{pb})$ at $\sqrt{\mathrm{s}}=1.96 \mathrm{TeV}$
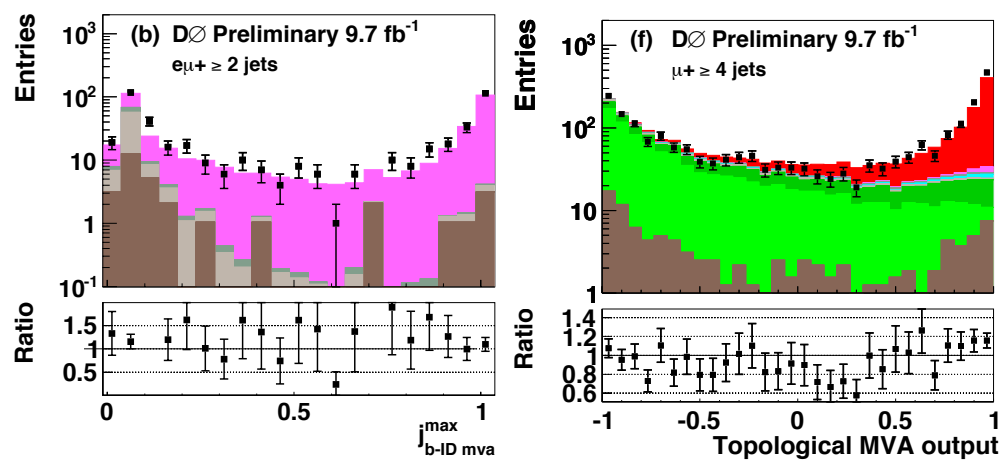

Figure 1: Summary of $t \bar{t}$ production cross section measurements at Tevatron, showing the most recent combination [3] (left), the $b$-tagging discriminator distribution in the D0 dilepton analysis [4] (centre) and the output variable of the multivariate analysis using topological variables in the $\ell+$ jets channel [4] (right).

The inclusive $t \bar{t}$ cross section has been determined in a variety of channels by ATLAS and CMS at 7 and $8 \mathrm{TeV}$. The $7 \mathrm{TeV}$ dataset is used by ATLAS to measure the branching ratios of topquark decays into leptons and jets [5]. Seven mutually exclusive final states are defined and used in the analysis, also including two channels with a lepton and a hadronically decaying $\tau$ lepton (Fig. 2 left).

At $8 \mathrm{TeV}$ a measurement in the single-lepton channel is presented by the ATLAS Collaboration, using events with at least three jets and at least one $b$-tagged jet [6]. Systematic uncertainties 
are smallest, when choosing only two discriminating input variables, the pseudorapidity of the lepton and the modified aplanarity, which are combined in a likelihood discriminant (Fig. 2 right).
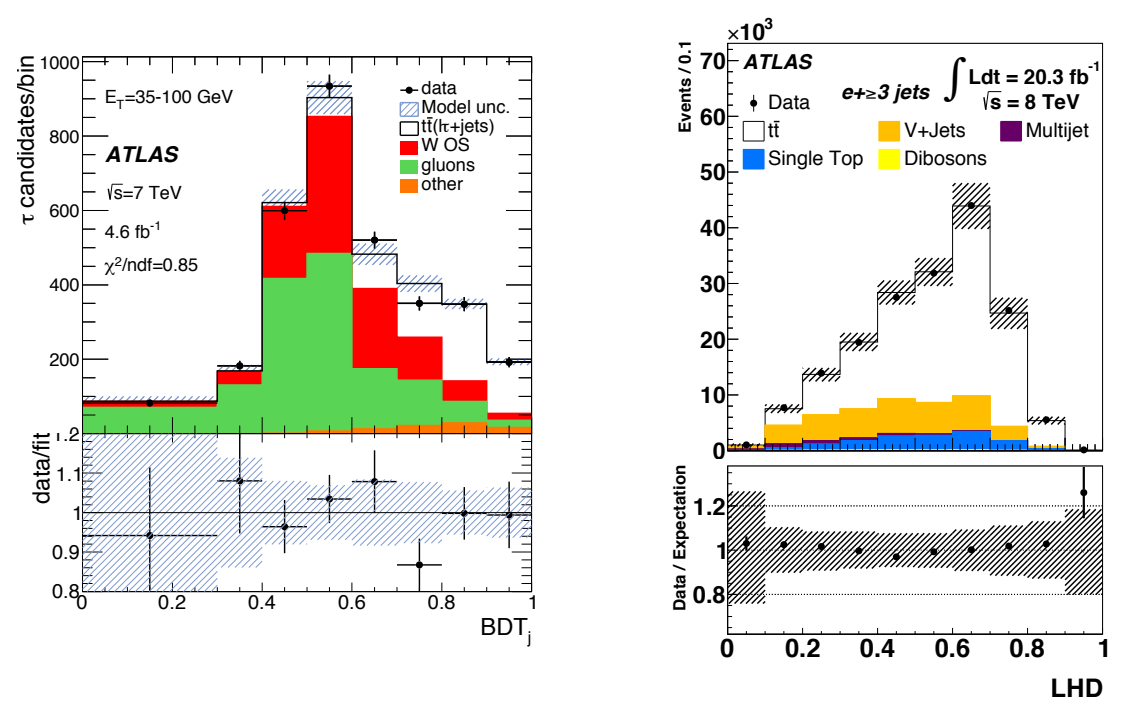

Figure 2: Left: Fitted distribution of the $\tau$-jet BDT discriminant for the $7 \mathrm{TeV} t \bar{t}$ cross-section determination [5]. Right: Likelihood discriminant in the $e+$ jets channel with at least three jets [6].

The best measurements of the inclusive $t \bar{t}$ cross section are performed in the dilepton $e \mu$ channel, as the background from multijet and vector-boson production can be reduced to a negligible level. Within the TOPLHCWG the best $8 \mathrm{TeV}$ measurements have been combined [7], allowing for a reduction of systematic uncertainties. The combined result is $\sigma_{t \bar{t}}=241.5 \pm 1.4$ (stat.) \pm 5.7 (syst.) \pm 6.2 (lumi.) pb, assuming a top-quark mass of $172.5 \mathrm{GeV}$. All measurements are compatible with the theoretical predictions (Fig. 3).
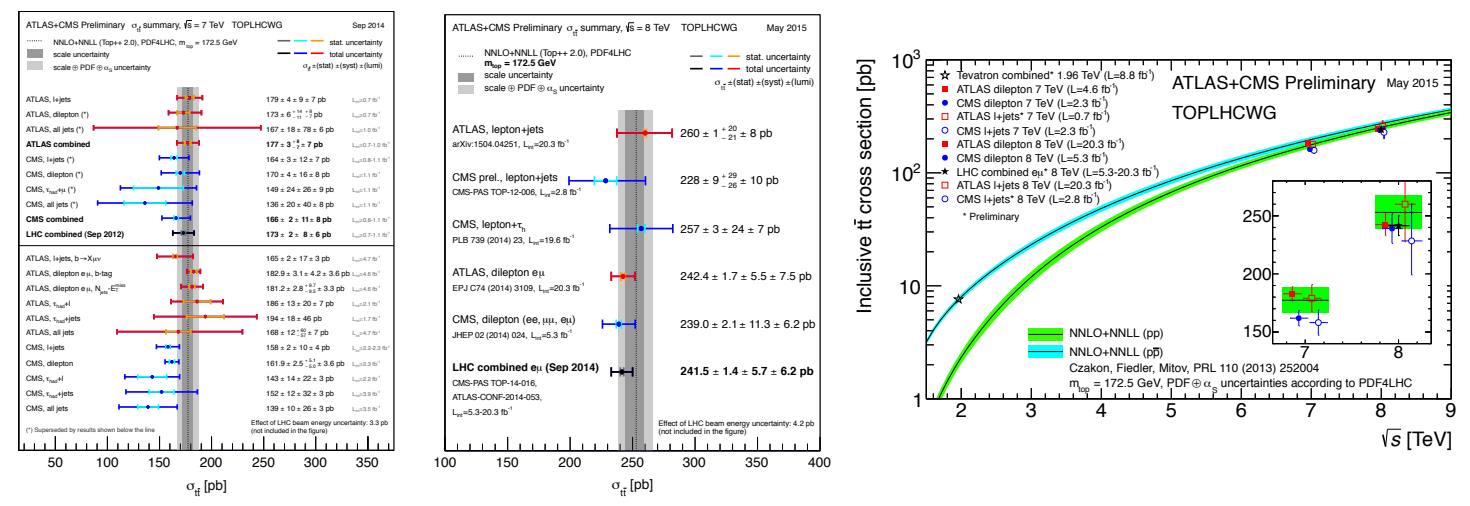

Figure 3: Summary of $t \bar{t}$ production cross-section measurements at the LHC [8] at $7 \mathrm{TeV}$ (left) and $8 \mathrm{TeV}$ (centre), and most precise measurements in each channel and experiment, together with the Tevatron and LHC combinations, as a function of the collision energy [8] (right). 
The LHCb experiment reported observation of top quarks in the forward region [9]. Albeit with large uncertainties this measurement is interesting in its own, as it probes a complementary region of phase space, possibly with enhanced sensitivity to new physics. The charge asymmetry and the number of events with a high- $p_{\mathrm{T}}$ muon and a $b$-tagged jet are compared, as a function of $p_{\mathrm{T}}$, to the hypothesis of $W b$ production (Fig. 4). The result is obtained in a fiducial region, with $p_{\mathrm{T}}(\mu)>25 \mathrm{GeV}, 2.0<\eta(\mu)<4.5, p_{\mathrm{T}}(b)>50 \mathrm{GeV}$ and $2.2<\eta(b)<4.2$. The likelihood fit in both variables shows that the background-only hypothesis is excluded at the $5.4 \sigma$ level. Fiducial $t \bar{t}$ cross sections are extracted at 7 and $8 \mathrm{TeV}$ with total uncertainties of $20-30 \%$ and found to be consistent with SM expectations.
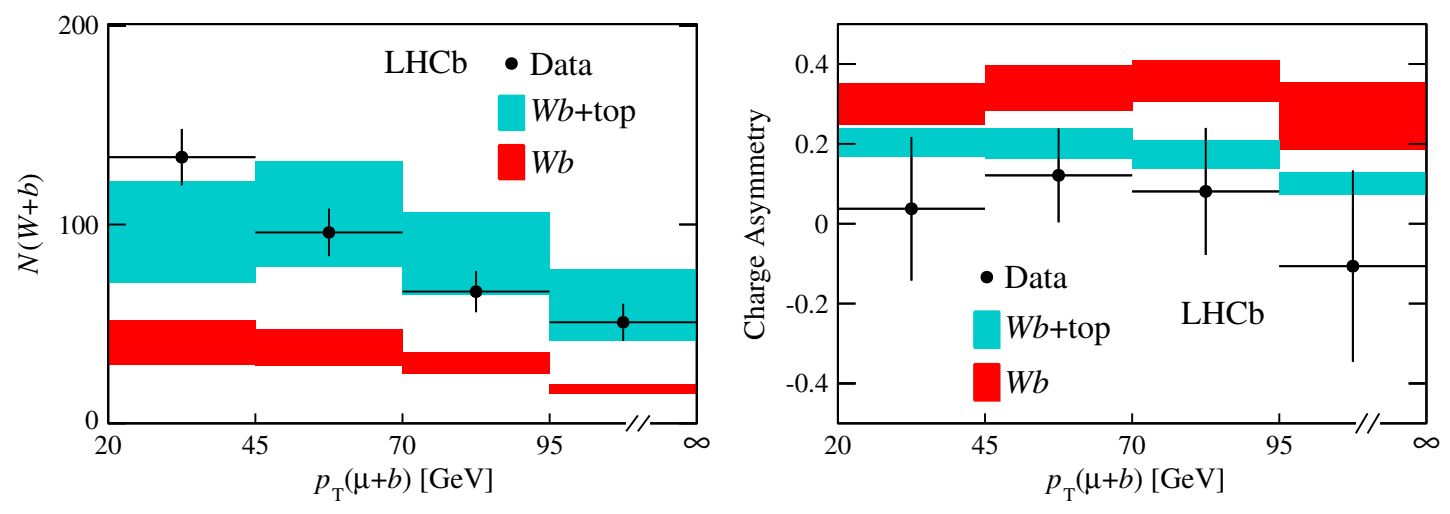

Figure 4: Observation of top quarks in the forward region [9]. Results for the $W b$ yield (left) and charge asymmetry (right) as a function of the transverse momentum of the $\mu b$-system, compared to data-constrained $W b$ and NLO top predictions.

ATLAS and CMS started analysing proton-proton collision data at $13 \mathrm{TeV}$, reporting first searches and measurements of Standard Model (SM) processes [10, 11]. Within few weeks of data-taking, events compatible with $t \bar{t}$ dilepton, single-lepton and single top quark production have been identified. With $\sim 80 \mathrm{pb}^{-1}$ ATLAS determines the $t \bar{t}$ inclusive cross section closely following the Run-1 strategy with $e \mu$ events [12]. The number of events with one or two $b$-tagged jets are used to simultaneously extract the cross section and the $b$-tagging efficiency. The CMS analysis employs this same channel, with a smaller dataset of $\sim 40 \mathrm{pb}^{-1}$, but without using the information of $b$-tagging and is based on a simple event counting technique [13]. Besides the uncertainty on the integrated luminosity, that affects the measurements at the level of 9-12\%, the most important systematic uncertainties are the modelling of the $t \bar{t}$ hadronisation for ATLAS (4.5\%) and the uncertainty deriving from the lepton triggers for CMS (5\%). The result of these first two measurements is (Fig. 5):

$$
\begin{aligned}
\sigma_{t \bar{t}}(\text { ATLAS }) & =825 \pm 49 \text { (stat.) } \pm 60 \text { (syst.) } \pm 83 \text { (lumi.) pb } \\
\sigma_{t \bar{t}}(\mathrm{CMS}) & =772 \pm 60 \text { (stat.) } \pm 62 \text { (syst.) } \pm 93 \text { (lumi.) pb. }
\end{aligned}
$$



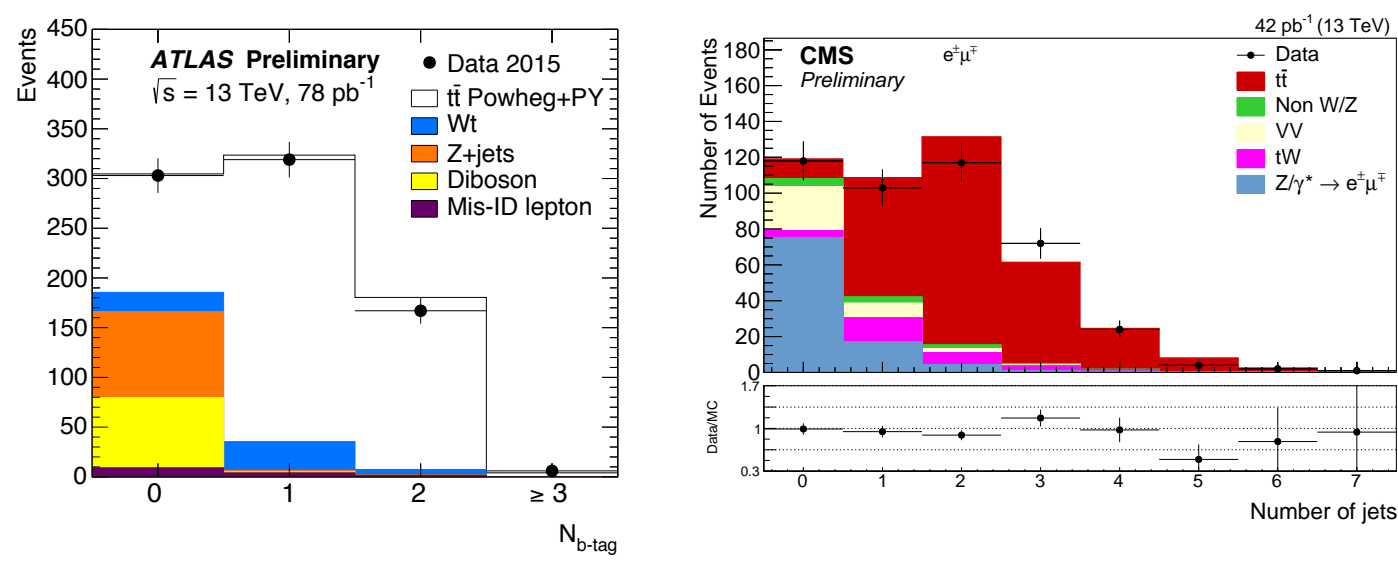

Figure 5: Measurement of the $t \bar{t}$ cross section at a centre-of-mass energy of $13 \mathrm{TeV}$. Number of $b$-tagged jets [12] (left) and number of jets [13] (right) for selected $e \mu$ events by ATLAS and CMS.

\section{Differential $t \bar{t}$ cross sections}

With the large top-quark sample available at the LHC it is now possible to study differential distributions. On one hand this allows for more detailed tests of perturbative QCD, to constrain the parton distribution functions (PDF) and the parameters of the Monte-Carlo simulation programs, on the other hand it allows for a better understanding of one of the major backgrounds in Higgs physics, rare processes or search for beyond SM (BSM) effects. The strategy for differential measurements is to start with a tight event selection to obtain a pure sample, enhanced with event reconstruction techniques. After the estimated background is subtracted, the effects of detector acceptance and resolution are removed by means of unfolding. Distributions are then presented at parton or particle level, as a function of kinematic quantities like $p_{\mathrm{T}}$ and $y$ of the $t \bar{t}$ system or of the top quarks, or of invariant masses.

A comprehensive set of top-quark differential measurements at $8 \mathrm{TeV}$ has been performed by CMS using final states with leptons [14]. The comparison to different matrix-element and partonshower programs is provided for the top-quark decay products, as well as for reconstructed objects at parton level. This analysis confirms the trend observed at $7 \mathrm{TeV}$ that the NLO simulation does not fully describe the $p_{\mathrm{T}}$ distribution of the top quark (Fig. 6).

Differential measurements presented at parton level are model dependent. In an effort to reduce theoretical uncertainties ATLAS made use of the concept of the so-called pseudo-top quark, a proxy object directly constructed from detector-level observables: charged leptons, jets and missing transverse momentum. The analysis of $7 \mathrm{TeV}$ data [15] reveals a good description of the data in general, with some discrepancies at low $m_{t \bar{t}}$ values. Within uncertainties the top- $p_{\mathrm{T}}$ distribution is sufficiently well described by PowHEG+PYTHIA (Fig. 7).

The high top-quark $p_{\mathrm{T}}$ region has been the target of further detailed studies, since a possible discrepancy could hint to physics effects beyond the Standard Model and studies allow for an improved understanding of the proton PDF. When top quarks are produced with large Lorentz boost their decay products tend to be more collimated and might escape standard reconstruction techniques, which exploit isolation. In particular, the hadronically decaying top quark is reconstructed 

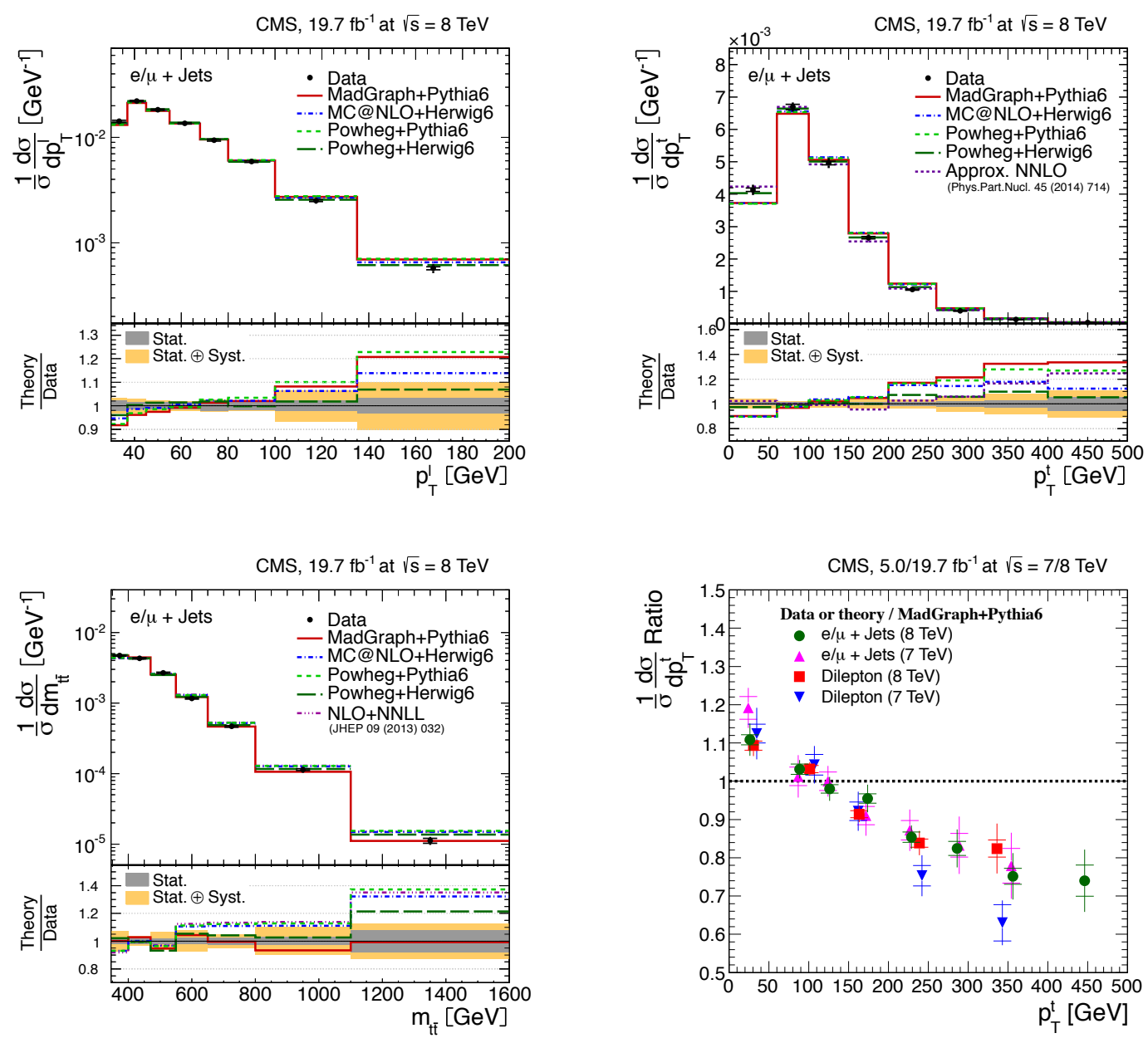

Figure 6: Normalised differential $t \bar{t}$ production cross section in the $\ell+$ jets channels compared to simulation [14], as a function of the transverse momentum of the lepton (top left) and of the reconstructed top quark (top right), and the invariant mass of the $t \bar{t}$ system (bottom left). Summary of all measurements as a function of top $p_{\mathrm{T}}[14]$ (bottom right).

as a single large-radius $(R)$ jet. Jet substructure techniques are employed to identify such large- $R$ jets and tested for compatibility with top-quark decays.

Measurements are performed with the full $8 \mathrm{TeV}$ dataset. ATLAS selects jets with $R=1$ and measures differential $t \bar{t}$ cross sections as a function of the top-quark $p_{\mathrm{T}}$ both, at particle level in a fiducial region closely following the event selection and at parton level [16]. The measurements have a threshold of $300 \mathrm{GeV}$ and extend beyond $1 \mathrm{TeV}$. Experimental uncertainties are of the order of $10-30 \%$ and are dominated by the jet energy scale uncertainty of large- $R$ jets. Discrepancies between data and simulations observed at low- $p_{\mathrm{T}}$ are in general confirmed in the boosted regime, although not statistically significant. Different PDF and parton-shower parameter settings can improve the agreement between data and simulation considerably (Fig. 8). CMS performs a similar analysis with $R=0.8$ jets to extract normalised differential distributions starting from $400 \mathrm{GeV}[17]$. 

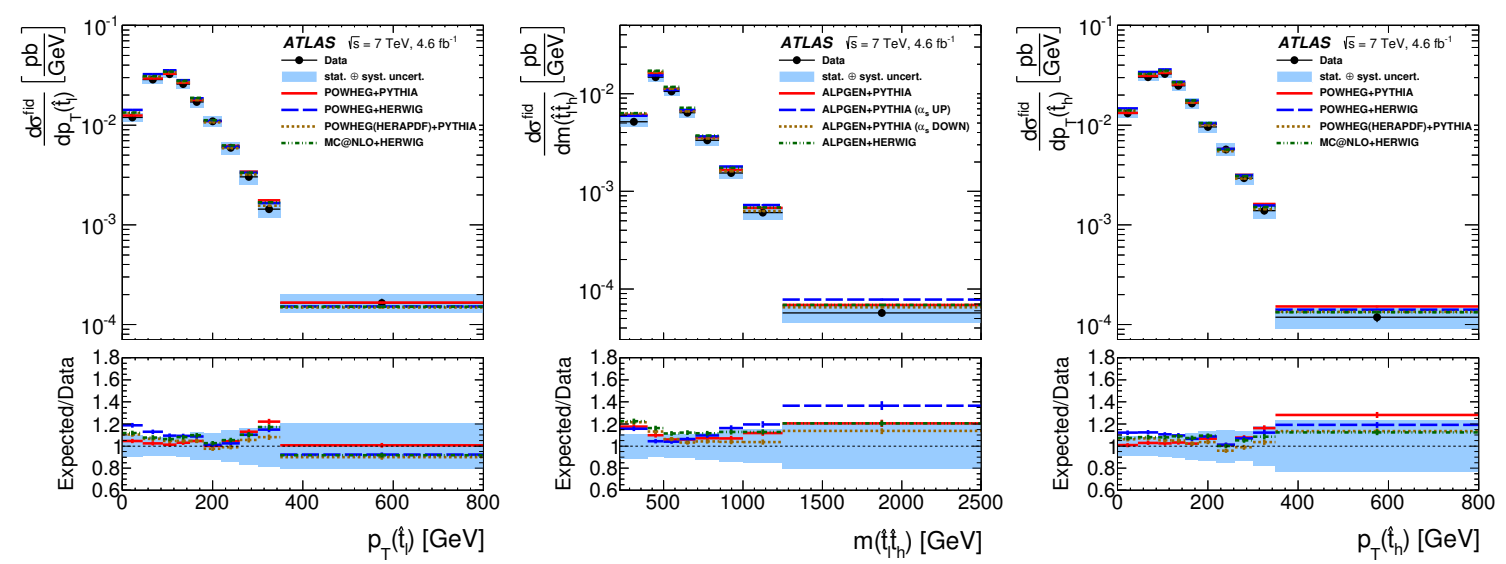

Figure 7: Differential $t \bar{t}$ cross section [15] as a function of the hadronic (left) and leptonic (centre) pseudotop-quark $p_{\mathrm{T}}$, and of the invariant mass of the two pseudo-top quarks (right). Model predictions from several generators are superimposed.
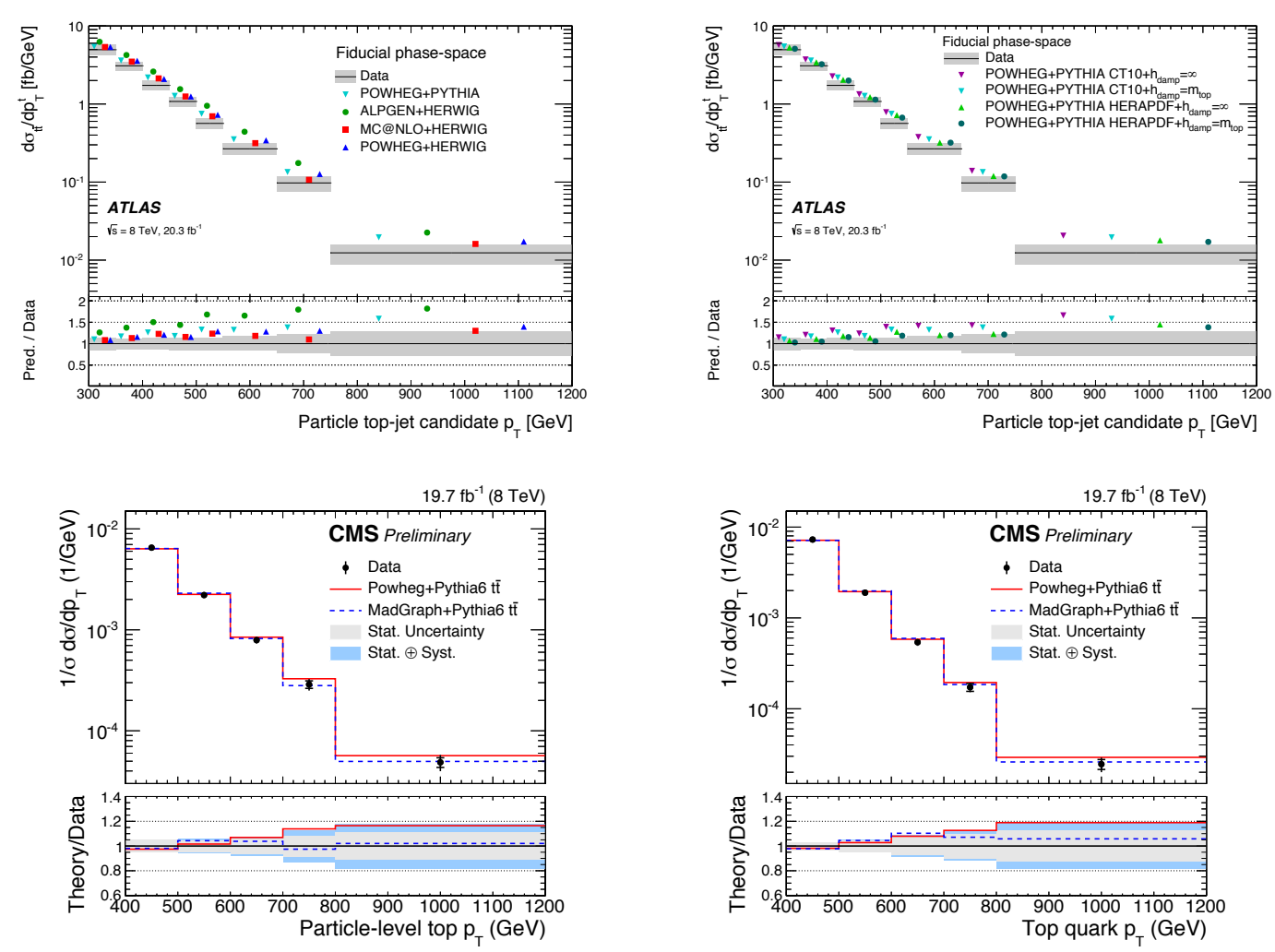

Figure 8: Fiducial particle-level differential cross section as a function of the hadronic top-jet candidate $p_{\mathrm{T}}$ reconstructed with boosted techniques [16], compared to LO and NLO generators interfaced to different parton-shower programs (top left) and to POWHEG+PYTHIA with varying shower parameter $h_{\text {damp }}$ or PDF (top right). Normalised differential cross section [17] in bins of the particle-level top-jet $p_{\mathrm{T}}$ (bottom left) and the parton-level top-quark $p_{\mathrm{T}}$ (bottom right). 


\section{Single-top quark production}

Besides the dominating pair-production of top quarks through the strong interaction, top quarks can also be produced singly via the weak vertex $W t b$. There are three possible single top-quark production modes at leading order in perturbation theory: an exchange of a virtual $W$ boson either in the $t$-channel or in the $s$-channel, or the associated production of a top-quark and a $W$ boson.

Much progress has been reported in the studies of single-top quark production in the three channels at Tevatron and LHC. In a final combination of the Tevatron datasets [18] the inclusive single-top $t$-channel cross section is measured with a relative uncertainty of $13 \%$. At the same time the $s$ - and $t$-channel production cross sections are simultaneously extracted in a two-dimensional measurement. A likelihood fit is performed to the binned distribution of a discriminant, optimised to separate signal events from large background contributions and to separate $s$-channel and $t$ channel events. The two channels are sensitive to different new physics effects, as can be seen in Fig. 9 and it is thus important to measure them separately. The $W t$ channel remains unaccessible at the Tevatron.
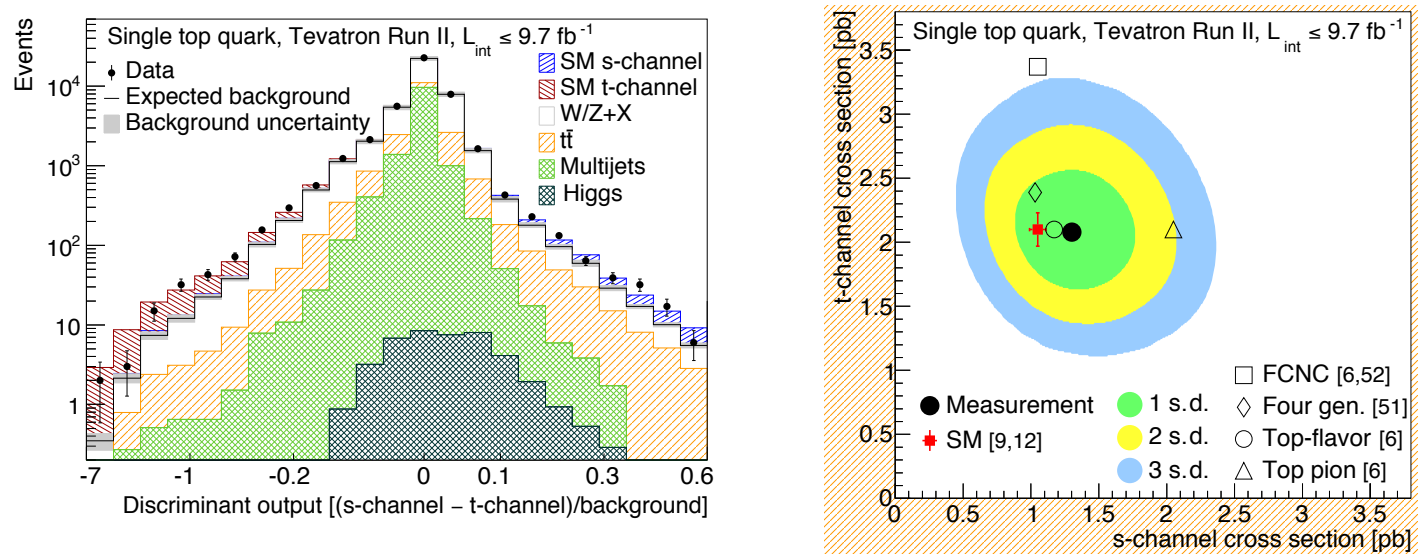

Figure 9: Distribution of the mean discriminants for bins with similar ratios of ( $s$-channel $-t$-channel) signals divided by background yields [18] (left) and two-dimensional posterior probability as a function of $\sigma_{t}$ and $\sigma_{s}$ with probability contours for the combination of the CDF and D0 analysis channels compared with the theoretical prediction of the SM and several BSM predictions [18] (right).

The large LHC dataset not only allows for precise measurements of the inclusive single top $t$-channel cross section at 7 and $8 \mathrm{TeV}$, but also for the measurement of properties and differential distributions. The most recent measurement is performed by the CMS Collaboration, employing a neural network (NN) discriminator with variables like the pseudorapidity of the light quark jet or the invariant mass of the reconstructed top-quark candidate $m_{\ell v b}$ [19]. After a stringent requirement on the NN discriminator output, a good ratio $S / \sqrt{S+B}$ is achieved and normalised background-subtracted and unfolded distributions as a function of the top-quark $p_{\mathrm{T}}$ and $|y|$ are shown to be in good agreement with NLO generators (Fig. 10), using four- or five-flavour schemes (aMC@NLO and PowheG), as well as $p_{\mathrm{T}}$-matched samples, that simulate $2 \rightarrow 2$ and $2 \rightarrow 3$ processes (COMPHEP).

At the LHC a measurement of the production in the $s$-channel is much more challenging, as it is suppressed by the requirement of a quark-antiquark pair in the colliding protons with sufficiently 

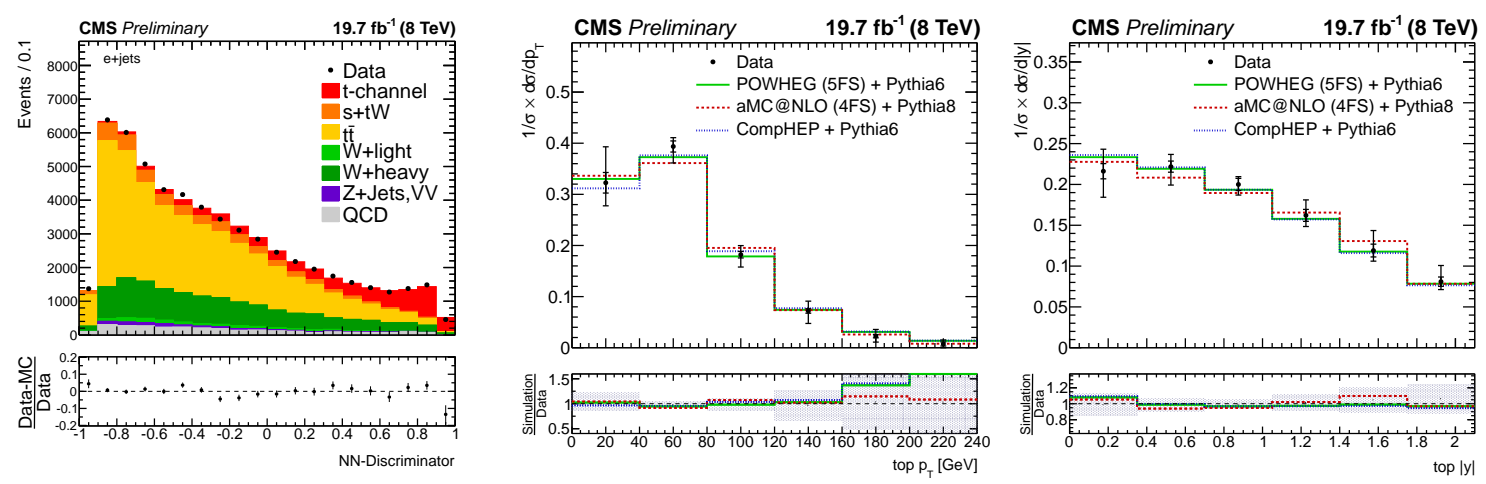

Figure 10: Measurement of the single top $t$-channel cross section at $8 \mathrm{TeV}$ [19]: neural-network output distribution in the $e+$ jets channel, requiring two jets and one $b$-tag (left), and unfolded spectra in the $\ell+$ jets channel compared with predictions as a function of top quark $p_{\mathrm{T}}$ (centre) and rapidity $|y|$ (right).

large $x$. Thus, while at Tevatron the expected ratio of $t$-to-s production is 2 , at $\mathrm{LHC}$ at $8 \mathrm{TeV}$ this ratio is 15 . Consequently the signal-to-background ratio in this channel is quite prohibitive at LHC. With the full dataset ATLAS performed a search for production in this channel [20]. After event selection with one lepton and exactly two jets, both of which are $b$-tagged, the signal-tobackground ratio is $3 \%$. A boosted decision tree (BDT) classifier is trained with kinematic and topological variables. The most discriminating variables are the differences in azimuthal angle $|\Delta \phi|$ between the $b$-jet and the top-quark candidate. The BDT output distribution is used to extract the signal contribution using a maximum likelihood fit (Fig. 11). The observed significance of the measurement is found to be $1.3 \sigma$ and the cross section is measured to be $\sigma_{s}=5.0 \pm 4.3 \mathrm{pb}$.
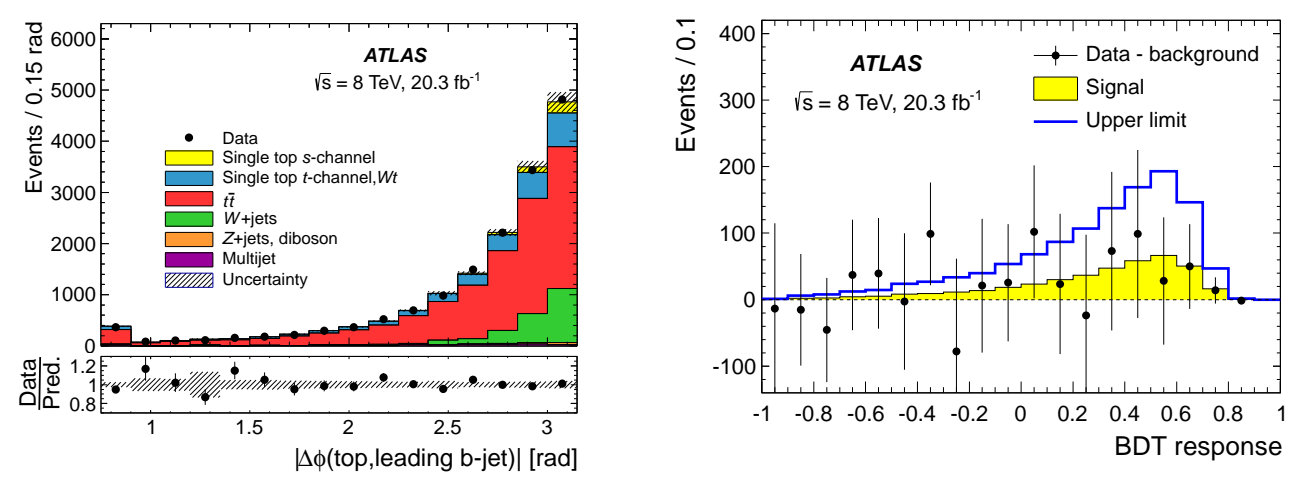

Figure 11: Measurement of the single top s-channel cross section at $8 \mathrm{TeV}$ [20]: observed and predicted distributions in the signal region for the azimuthal angle between the leading $b$-jet and the top-quark candidate reconstructed with the sub-leading $b$-jet (left), and BDT response after background subtraction (right).

Measurements of single top production in the $W t$ channel at the LHC have been combined within the TOPLHCWG [21]. In both cases the dilepton signature with one or two jets is exploited as it offers a good signal-to-background ratio. The final discrimination is obtained with BDTs. The CMS measurement uses all dilepton channels and a partial dataset, while the ATLAS measurement employs only the $e \mu$ channel and the full dataset. The total uncertainty of $23 \%$ in each of the input 
measurements is reduced to $19 \%$ in the combination, with small statistical uncertainties (Fig. 12). The uncertainty due to the matching of matrix element and parton shower and the choice of scales dominate the systematic uncertainty.

Each measurement of single top production is in good agreement with the Standard Model expectation (Fig. 12) and can be separately interpreted in terms of a determination of the CKM matrix element $\left|V_{t b}\right|$. The Tevatron combination extracts a value of $1.02_{-0.05}^{+0.06}$, while at the LHC the measurements with the smallest uncertainties are derived from the CMS 7 and $8 \mathrm{TeV}$ combination for the $t$-channel $(1.00 \pm 0.04)$ and the LHC combination for the $W t$ channel $(1.06 \pm 0.11)$.
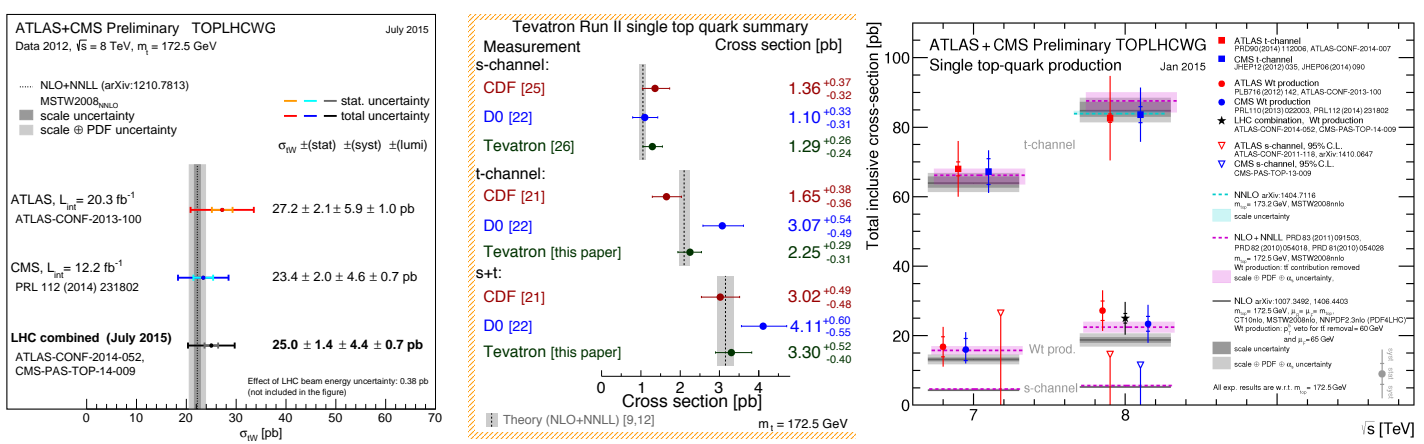

Figure 12: Single top summary plots including the results of combinations and compared to predictions: TOPLHCWG $8 \mathrm{TeV} W t$ channel combination [21] (left), Tevatron Run II [18] (centre) and LHC summary plot [8] (right).

\section{Associated production}

Several measurements of top-quark production in association with further particles have been performed. Recent observation, evidence or search for production of top quark pairs in association with photons, electroweak bosons, as well as heavy quarks have been reported and are discussed in the following. The production of $t \bar{t} H$ is presented elsewhere [22].

The production cross section of top-quark pairs with additional photons is sensitive to the $t \bar{t} \gamma$ coupling and a measurement can be used to constrain new physics, for instance with composite or excited top quarks. With the full $7 \mathrm{TeV}$ dataset the ATLAS Collaboration reports observation of this process with a significance of $5.3 \sigma$ [23]. The measurement is performed in the single-lepton channel in a fiducial region, requiring an identified photon with $E_{T}>20 \mathrm{GeV}$, isolated from jets and from leptons, in order to suppress the contribution of photons not radiated from top quarks. The cross section is extracted from a template fit to a variable that measures the track activity near the photon candidate $\left(p_{\mathrm{T}}^{\mathrm{iso}}\right)$ and discriminates between signal photons and neutral hadron decays or misidentified photons (Fig. 13). With 362 selected events the fiducial cross section is measured with an uncertainty of $\sim 30 \%$, dominated by uncertainties on the jet energy scale and $b$-tagging efficiency.

At energies and luminosities available at the LHC the associated production of top-quark pairs with heavy vector bosons $\left(W\right.$ or $Z$ ) become accessible. The production cross sections $\sigma_{t \bar{t} W}$ and $\sigma_{t \bar{Z} Z}$ are simultaneously extracted in channels with two or more leptons, since the two processes are 

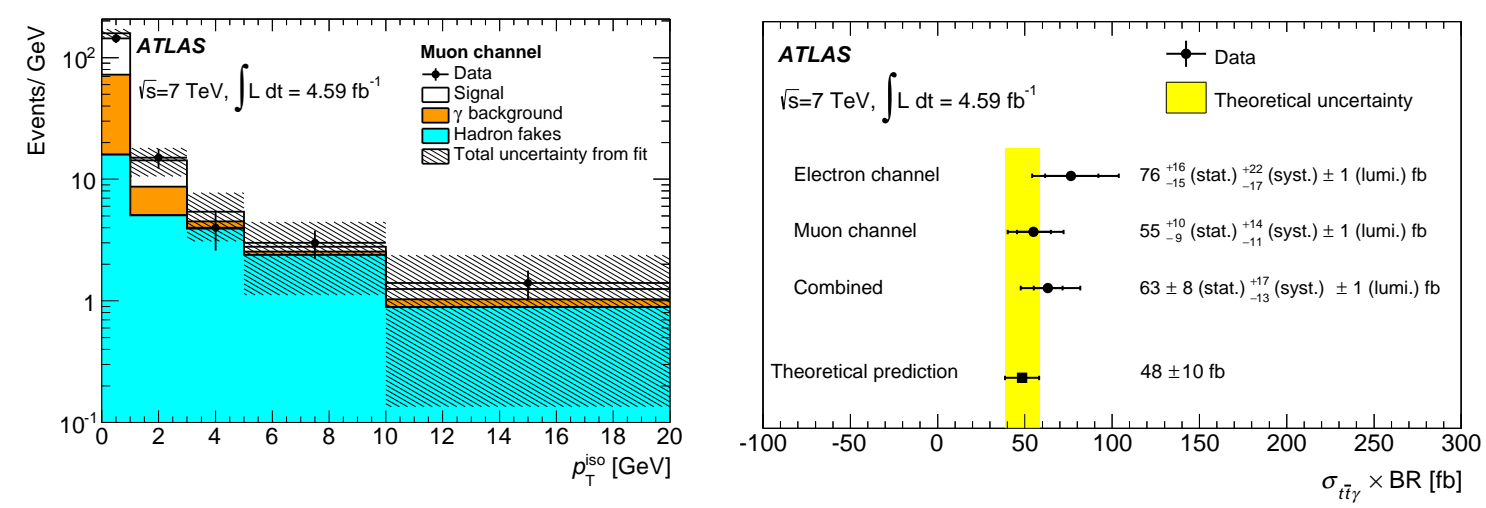

Figure 13: Measurement of the $t \bar{t} \gamma$ cross section at $7 \mathrm{TeV}$ [23]: results of the combined likelihood fit using the track-isolation $\left(p_{\mathrm{T}}^{\text {iso }}\right)$ distribution as the discriminating variable in the muon channel (left) and summary of results compared to the theoretical prediction in the fiducial region (right).

experimentally intertwined. The interest in the measurement of the $t \bar{t} Z$ process lies in the determination of the top-quark coupling to the $Z$ boson. The $t \bar{t} W$ process probes the proton structure and is a source of same-sign dilepton events, which is an important background in many searches. A variety of new physics models can alter the prediction of $\sigma_{t \bar{t} W}$ and $\sigma_{t \bar{t} Z}$ and their effects are typically parameterised by dimension-six operators in an effective field theory.

Using four signatures (opposite-sign dilepton, same-sign dilepton, trilepton, and tetralepton) and performing a simultaneous maximum likelihood fit, ATLAS extracts both cross sections with a significance of $5.0 \sigma$ and $4.2 \sigma$, respectively, over the background-only hypothesis [24]. In the opposite-sign dilepton channel the signal-to-background ratio is particularly challenging and therefore control regions to determine the most important backgrounds from data are included in the fit and a neural network is employed in the signal regions. In the same-sign dilepton channel the contribution of fake leptons and charge misidentification is carefully studied. The tri- and tetralepton channels effectively measure the $t \bar{t} Z$ contribution. Important irreducible Standard Model backgrounds that produce three or four leptons are diboson $(W Z, Z Z)$ events, determined through fits in control regions, and associated single top quark production $(t Z, W t Z)$. The measured cross sections are $\sigma_{t \bar{t} W}=369_{-91}^{+100} \mathrm{fb}$ and $\sigma_{t \overline{t Z}}=176_{-52}^{+58} \mathrm{fb}$, with uncertainties dominated by the statistical component (Fig. 14 top).

CMS determines the cross sections of these processes, as well, with significances of $4.8 \sigma$ and $6.4 \sigma$, respectively [25], using the same final states. The analysis strategy aims at reducing the statistical uncertainty by lowering the requirements on the reconstructed objects quality, at the expense of larger systematic uncertainties. A full reconstruction of pre-selected events is attempted, by matching the reconstructed objects in the detector to the decaying $W$ and $Z$ bosons, and to the top quark. A linear discriminant helps to determine the best permutation in matching jets and leptons. Signal is separated from background by means of several BDTs, one in each channel and jet multiplicity, which are trained with this discriminant and other kinematic quantities (Fig. 14 bottom). The measured cross sections are $\sigma_{t \overline{ } W}=382_{-102}^{+117} \mathrm{fb}$ and $\sigma_{t \bar{t} Z}=242_{-55}^{+65} \mathrm{fb}$. The result is then used to place constraints on the axial and vector components of the $t \bar{t} Z$ coupling and on dimension-six operators in an effective field theory framework. 

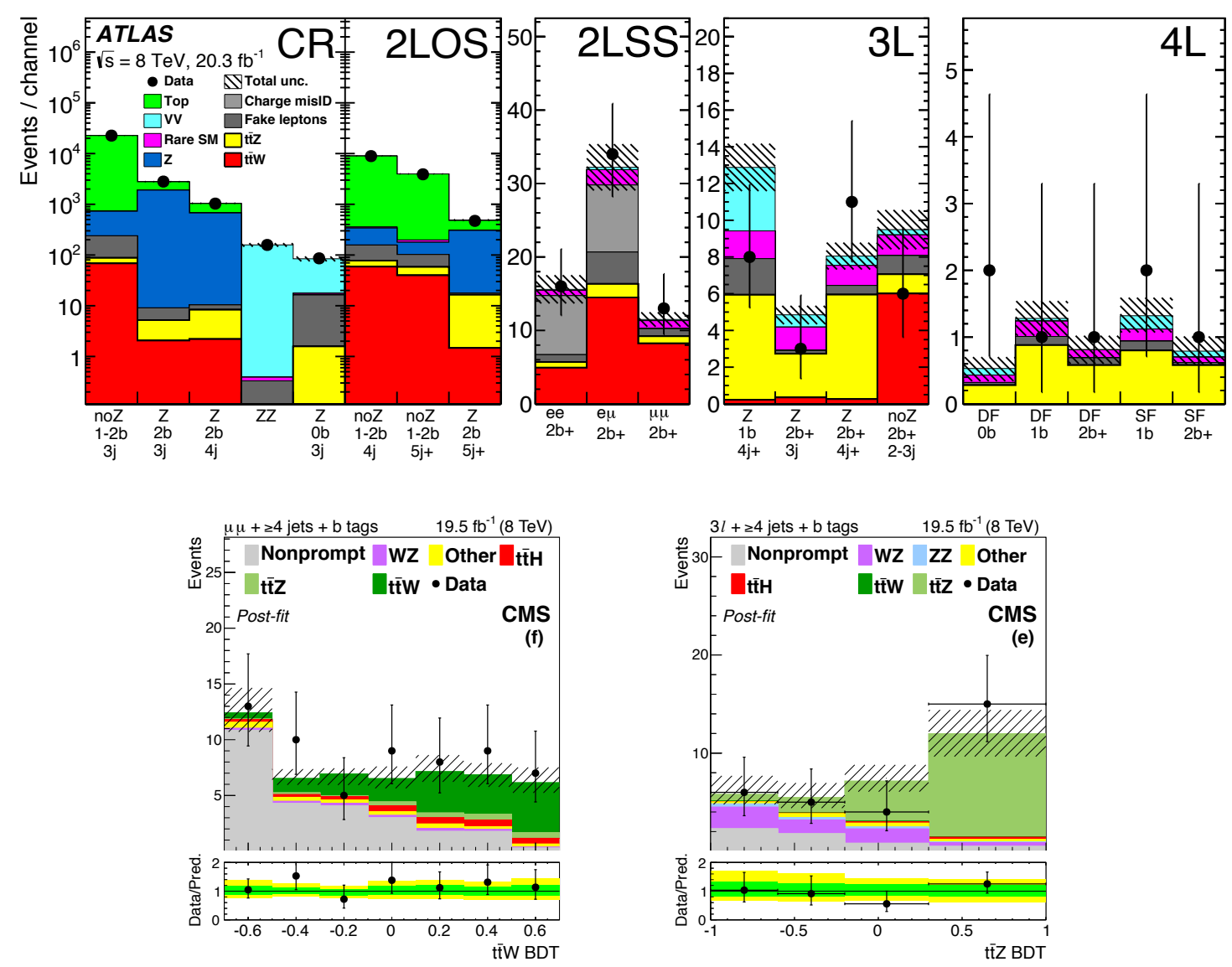

Figure 14: Measurement of the $t \bar{t} W$ and $t \bar{t} Z$ cross sections at $8 \mathrm{TeV}$. Expected yields after the fit compared to data in the four signal channels and five control regions (CR) used to constrain the $t \bar{t}, Z, Z Z$ and $W Z$ backgrounds [24] (top), and final discriminants for the $t \bar{t} W$ BDT in the same-sign $\mu \mu$ channel (bottom left) and the $t \bar{t} Z$ BDT in the trilepton channel (bottom right), both in events with at least four jets [25].
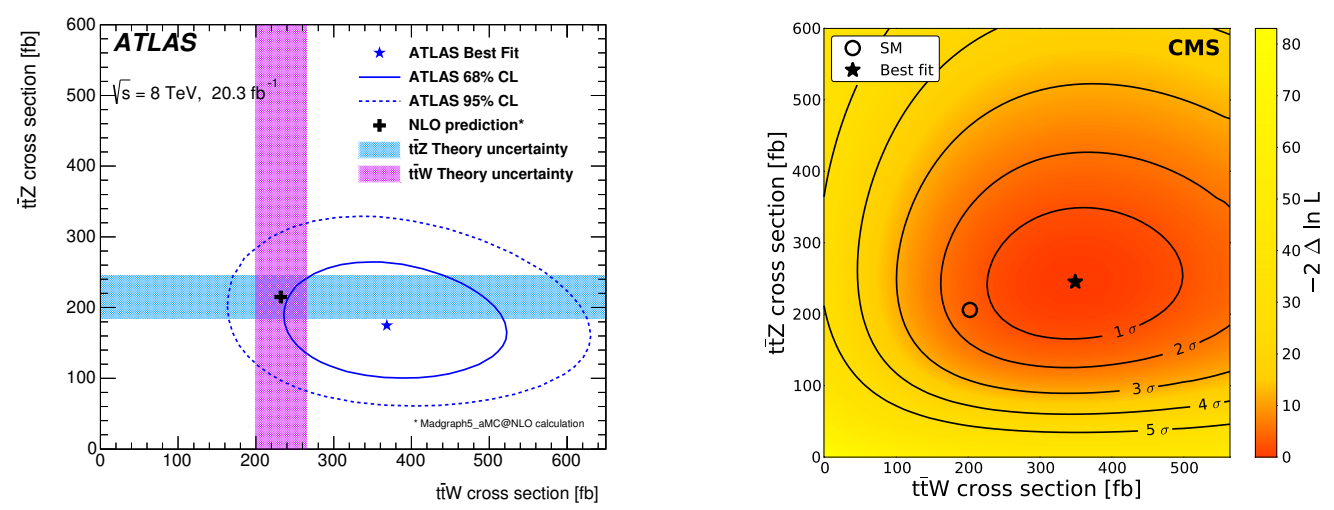

Figure 15: Result of the simultaneous fit to the $t \bar{t} W$ and $t \bar{t} Z$ cross sections along with uncertainty contours compared to theoretical predictions, for the ATLAS [24] (left) and CMS [25] (right) measurements. 


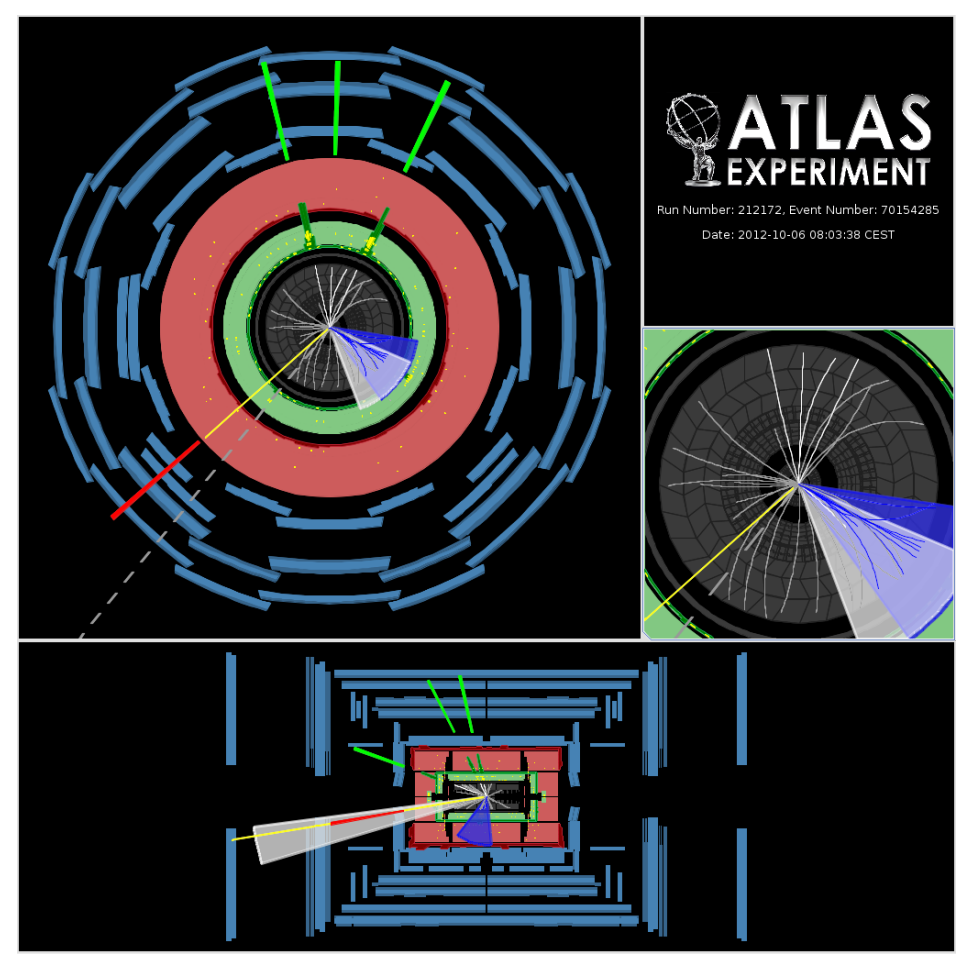

Figure 16: A display of a $t \bar{t} Z$ candidate in the tetralepton channel [24]. The event features a pair of electrons (green) with $m_{e e}=93 \mathrm{GeV}$, and additionally a muon (red) and an extra electron, as well as two jets (blue and white cones), both of which are $b$-tagged. The missing transverse energy $\left(E_{\mathrm{T}}^{\mathrm{miss}}=57 \mathrm{GeV}\right)$ is represented by the grey dashed line.

Top-quark pairs are often produced with additional energetic jets. The measurement of such jet multiplicities provides an important test of the QCD predictions at NLO. Recently, the production of additional $b$-quarks has been studied. The $t \bar{t} b \bar{b}$ final state is an irreducible non-resonant background to the $t \bar{t} H$ process and difficult to model in simulation because of ambiguities in the matching to the parton shower.

CMS measures the total cross section $\sigma_{t \bar{t} b \bar{b}}$ and the quantity $R_{\mathrm{HF}}=\frac{\sigma_{t \bar{b} \bar{b}}}{\sigma_{t \bar{t} j}}$ in the dilepton [26] and single-lepton channels [27]. In the first case, events with two well identified $b$-jets and at least two additional jets are required. The $b$-tagging algorithm discriminator of the third and fourth jet are used to separate $t \bar{t} b \bar{b}$ events from the background, including $t \bar{t} j j$, with a template fit (Fig. 17). For the measurement in the single-lepton channel the jets from the top-quark decay are identified using a constrained kinematic fit and multivariate classifiers in different categories split by the jet multiplicity. Results range in $R_{\mathrm{HF}}=0.012-0.022$, depending on the phase space and definition considered (particle or parton level) with uncertainties of $0.004-0.006$, and are in general in good agreement with predictions.

ATLAS performs four measurements of heavy flavour production in top-quark pair events [28] in a fiducial volume: a fit-based and a cut-based measurement of $\sigma_{t \bar{t} b \bar{b}}$ in the dilepton channel, and cut-based measurements of $\sigma_{t \bar{t} b}$ in the dilepton and in the single-lepton channels. The ratio $R_{\mathrm{HF}}$ is determined to be $0.013 \pm 0.004$ with comparable systematic and statistical uncertainties. The cutbased analysis uses very tight selection criteria, including the requirement of four $b$-tagged jets, 
relies on simulation for the background determination and features a high signal-to-background ratio. A looser selection is applied in the second analysis where the signal is extracted from a fit to the multivariate $b$-jet identification discriminant. More events are produced in the single-lepton channel, however this channel is affected by additional backgrounds, where a $W$ boson can produce a $c$ quark. The measurements are also presented after subtracting the expected contributions from electroweak processes $(t \bar{t} W, t \bar{t} Z$ and $t \bar{t} H)$ in order to allow for comparison with NLO QCD theory predictions. They are then compared to predictions using different $g \rightarrow b \bar{b}$ splitting in the parton shower. The most extreme PyTHIA 8 model is disfavoured by the measurements (Fig. 17).
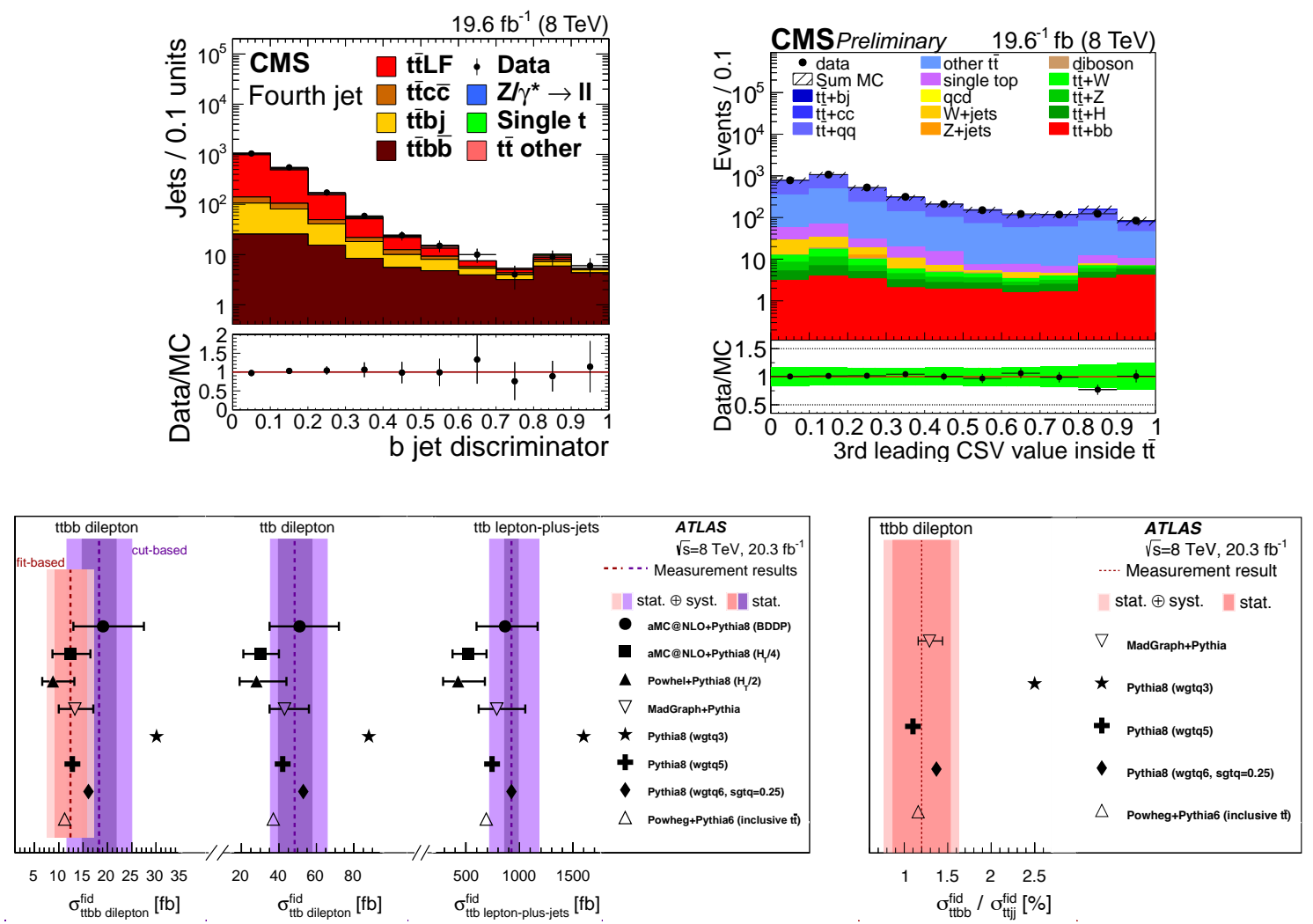

Figure 17: Measurements of $t \bar{t}$ with heavy flavour quarks at $8 \mathrm{TeV}$. Distribution of the $b$-jet discriminator for the fourth jet in decreasing order of $b$-tagging discriminator value in the dilepton channel [26] (top left), pre-fit distribution of the $b$-tag discriminant of the third jet in the $\mu+$ jets channel for events with five jets [27] (top right), comparison of the measured cross sections in the three fiducial regions with theoretical predictions [28] (bottom left) and comparison of the measured ratio $R_{\mathrm{HF}}$ with different generator settings after subtraction of electroweak contributions [28] (bottom right).

The production of four top quarks is a rare process in the Standard Model, proceeding via gluon-gluon fusion or quark-antiquark annihilation and is predicted to have a total cross section of $\sim 1 \mathrm{fb}$ at $8 \mathrm{TeV}$. The production can be significantly enhanced in BSM physics models and therefore ATLAS and CMS searched for evidence of production of this process. In a dedicated search [29] CMS selects events with one lepton, at least six jets and two $b$-tagged jets with large $H_{\mathrm{T}}$. Kinematic reconstruction techniques and multivariate discriminants are employed to place an upper limit on the production cross section. ATLAS analyses events with a pair of leptons with 
the same charge with at least one $b$-tagged jet and large $H_{\mathrm{T}}$ [30] to search for enhancements in the four-top production or evidence of new physics. A search for vector-like quarks in the single-lepton channel by ATLAS [31] is reinterpreted and used to set the best limit, $\sigma_{t \bar{t} t \bar{t}}<23 \mathrm{fb}$.

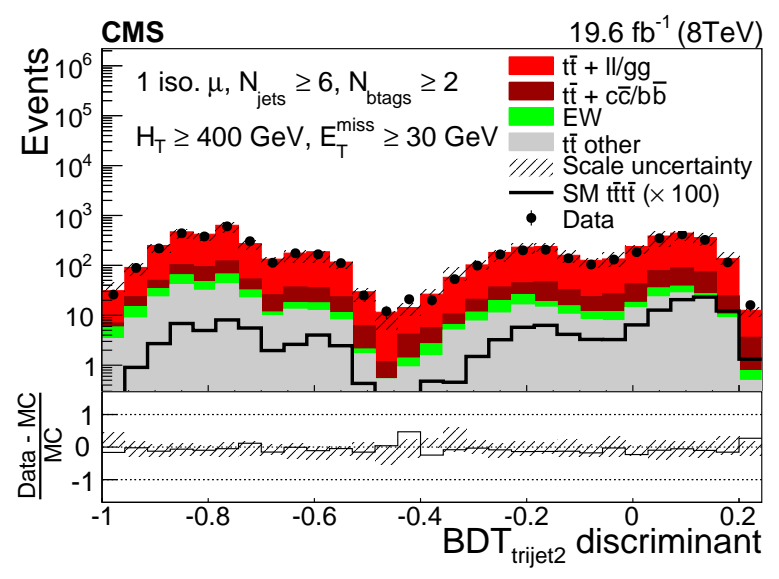

Figure 18: Search for $t \bar{t} t \bar{t}$ production: Distribution of the BDT discriminant aiming at identifying a second hadronically decaying top quark in the single-lepton channel [29].

\section{Summary and conclusion}

Top-quark production measurements provide stringent tests of perturbative QCD and have reached a good level of maturity with the large data samples available. The inclusive top-quark pair production is measured at centre-of-mass energies of 1.96, 7 and $8 \mathrm{TeV}$, with an uncertainty of 4-5\%, a better accuracy than the calculations at NNLO+NNLL. First measurements of the inclusive cross section are also available in the forward region and at $13 \mathrm{TeV}$, albeit with larger uncertainties. Differential measurements allow to test in more depth the validity of the calculations and the simulation programs that are used at hadron colliders. Results are reported after unfolding for detector effects, extrapolating to either the parton or particle level. The high- $p_{\mathrm{T}}$ region is also explored, where top-quark decay products start to merge and so-called boosted techniques improve the reconstruction efficiency. In general, data and prediction match well, with some discrepancies that might arise from the limited accuracy in the calculations used in the simulation (NLO). Single-top quark production has now been observed in the $t$ - and $s$-channels separately at Tevatron, while at the LHC the $W t$ channel has been observed in a combination and the abundant $t$-channel allows for differential measurements. The CKM matrix element $\left|V_{t b}\right|$ is determined with an uncertainty of $4 \%$ and is compatible with unity. Associated production of top-quark pairs with additional bosons or quarks have smaller production cross sections and start to become accessible with the Run-1 dataset of LHC. Production of $t \bar{t} \gamma, t \bar{t} W$ and $t \bar{t} Z$ have all been observed, while upper limits are placed on $t \bar{t} t \bar{t}$. Understanding additional heavy-flavour production is important, for instance as a background to $t \bar{t} H$, and is therefore matter of detailed study. With the upcoming Run-2 dataset, to be collected at $13 \mathrm{TeV}$ in the next few years, it will be possible to challenge current calculations and simulations at a next level of precision. 


\section{Acknowledgements}

The work of the author is currently funded by the European Research Council under the European Union's Seventh Framework Programme ERC Grant Agreement n. 617185.

\section{References}

[1] K. Melnikov, Theoretical Results on Top Quark Physics, talk at this conference, https://indico.cern.ch/event/325831/session/10/contribution/30.

[2] A. Meyer, Determination of Top Quark Properties, talk at this conference, https://indico.cern.ch/event/325831/session/10/contribution/32.

[3] T. Aaltonen et al. (CDF and D0 Collaborations), Combination of measurements of the top-quark pair production cross section from the Tevatron Collider, Phys. Rev. D 89 (2014) 072001.

[4] D0 Collaboration, Measurement of the inclusive t $\bar{t}$ production cross section in $p \bar{p}$ collisions at $\sqrt{s}=1.96 \mathrm{TeV}$, D0 Note 6453-CONF, www-d0.fnal.gov/Run2Physics/WWW/results/prelim/TOP/T106.

[5] ATLAS Collaboration, Measurements of the top quark branching ratios into channels with leptons and quarks with the ATLAS detector, Phys. Rev. D 92 (2015) 072005.

[6] ATLAS Collaboration, Measurement of the top pair production cross section in 8 TeV proton-proton collisions using kinematic information in the lepton+jets final state with ATLAS, Phys. Rev. D 91 (2015) 112013.

[7] ATLAS and CMS Collaborations, Combination of ATLAS and CMS top quark pair cross section measurements in the e $\mu$ final state using proton-proton collisions at $\sqrt{\mathrm{s}}=8 \mathrm{TeV}$, ATLAS-CONF-2014-054, http://inspirehep.net/record/1319552, CMS PAS TOP-14-016, http://inspirehep.net/record/1319376.

[8] ATLAS and CMS Collaborations, TOPLHCWG summary plots, twiki.cern.ch/twiki/bin/view/LHCPhysics/TopLHCWGSummaryPlots.

[9] LHCb Collaboration, R. Aaij et al., First observation of top quark production in the forward region, Phys. Rev. Lett. 115 (2015) 112001.

[10] B. Heinemann, ATLAS Results from Run2, talk at this conference, https://indico.cern.ch/event/325831/session/0/contribution/8.

[11] L. Malgeri, CMS Results from Run2, talk at this conference, https://indico.cern.ch/event/325831/session/0/contribution/9.

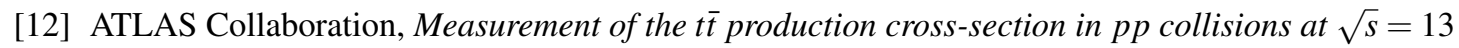
TeV using e $\mu$ events with b-tagged jets, ATLAS-CONF-2015-033, http://inspirehep.net/record/1385192.

[13] CMS Collaboration, Measurement of the top quark pair production cross section in proton-proton collisions at $\sqrt{s}=13 \mathrm{TeV}$, CMS PAS TOP-15-003, http://inspirehep.net/record/1388254.

[14] CMS Collaboration, Measurement of the differential cross section for top quark pair production in pp collisions at $\sqrt{s}=8 \mathrm{TeV}$, submitted to Eur. Phys. J. C, arXiv:1505.04480 [hep-ex].

[15] ATLAS Collaboration, Differential top-antitop cross-section measurements as a function of observables constructed from final-state particles using pp collisions at $\sqrt{s}=7 \mathrm{TeV}$ in the ATLAS detector, JHEP 06 (2015) 100. 
[16] ATLAS Collaboration, Measurement of the differential cross-section of highly boosted top quarks as a function of their transverse momentum in $\sqrt{s}=8 \mathrm{TeV}$ proton-proton collisions using the ATLAS detector, submitted to PRD, arXiv:1510.03818 [hep-ex].

[17] CMS Collaboration, Measurement of the differential t $\bar{t}$ production cross section for high- $p_{\mathrm{T}}$ top quarks in e/ $\mu+$ jets final states at $\sqrt{s}=8 \mathrm{TeV}$, CMS PAS TOP-14-012, http://inspirehep.net/record/1388555.

[18] T. Aaltonen et al. (CDF and D0 Collaborations), Tevatron combination of single-top-quark cross sections and determination of the magnitude of the Cabibbo-Kobayashi-Maskawa matrix element $V_{t b}$, Phys. Rev. Lett. 115 (2015) 152003.

[19] CMS Collaboration, Measurements of the differential cross section of single top-quark production in the $t$ channel in proton-proton collisions at $\sqrt{s}=8 \mathrm{TeV}$, CMS PAS TOP-14-004, http://inspirehep.net/record/1323200.

[20] ATLAS Collaboration, Search for s-channel single top-quark production in proton-proton collisions at $\sqrt{s}=8 \mathrm{TeV}$ with the ATLAS detector, Phys. Lett. B 740 (2015) 118.

[21] ATLAS and CMS Collaborations, Combination of cross-section measurements for associated production of a single top-quark and $a \mathrm{~W}$ boson at $\sqrt{s}=8 \mathrm{TeV}$ with the ATLAS and CMS experiments, ATLAS-CONF-2014-052, http://inspirehep.net/record/1319379 and CMS PAS TOP-14-009, http://inspirehep.net/record/1319686.

[22] S. Farrington, Results on the Standard Model Higgs boson, talk at this conference, https://indico.cern.ch/event/325831/contribution/11.

[23] ATLAS Collaboration, Observation of top-quark pair production in association with a photon and measurement of the $t \bar{t} \gamma$ production cross section in pp collisions at $\sqrt{s}=7$ TeVusing the ATLAS detector, Phys. Rev. D 91 (2015) 072007.

[24] ATLAS Collaboration, Measurement of the $t \bar{t} W$ and $t \bar{t} Z$ production cross sections in pp collisions at $\sqrt{s}=8 \mathrm{TeV}$ with the ATLAS detector, accepted by JHEP, arXiv:1509.05276 [hep-ex].

[25] CMS Collaboration, Observation of top quark pairs produced in association with a vector boson in pp collisions at $\sqrt{s}=8 \mathrm{TeV}$, submitted to JHEP, arXiv:1510.01131 [hep-ex].

[26] CMS Collaboration, Measurement of the cross section ratio $\sigma_{t \bar{t} b \bar{b}} / \sigma_{t \bar{t} j j}$ in pp collisions at $\sqrt{s}=8 \mathrm{TeV}$, Phys. Lett. B 746 (2015) 132.

[27] CMS Collaboration, Measurement of the $t \bar{t} b \bar{b}$ cross section and the ratio $\sigma(t \bar{t} b \bar{b}) / \sigma(t \bar{t} j j)$ in the lepton+jets final state at $8 \mathrm{TeV}$ with the CMS detector, CMS PAS TOP-13-016, http://inspirehep.net/record/1385669.

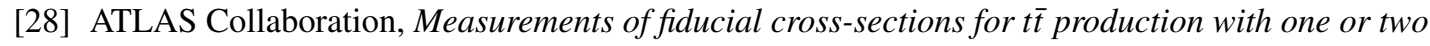
additional b-jets in pp collisions at $\sqrt{s}=8 \mathrm{TeV}$ using the ATLAS detector, submitted to Eur. Phys. J. C, arXiv:1508.06868 [hep-ex].

[29] CMS Collaboration, Search for standard model production of four top quarks in the lepton + jets channel in pp collisions at $\sqrt{s}=8 \mathrm{TeV}$, JHEP 11 (2014) 154 .

[30] ATLAS Collaboration, Analysis of events with b-jets and a pair of leptons of the same charge in pp collisions at $\sqrt{s}=8 \mathrm{TeV}$ with the ATLAS detector, JHEP 10 (2015) 150.

[31] ATLAS Collaboration, Search for production of vector-like quark pairs and of four top quarks in the lepton-plus-jets final state in pp collisions at $\sqrt{s}=8 \mathrm{TeV}$ with the ATLAS detector, JHEP 08 (2015) 105. 